\title{
Eps8 promotes cellular growth of human malignant gliomas
}

\author{
XIAOFENG DING $^{1 *}$, FANGLIANG ZHOU $^{1,2^{*}}$, FANGMEI WANG $^{1}$, ZIJIAN YANG $^{1}$, \\ CHANG ZHOU $^{1}$, JIANLIN ZHOU ${ }^{1}$, BO ZHANG ${ }^{1,2}$, JUNMEI YANG ${ }^{3}$, GUANGWEI WANG ${ }^{1,3}$, \\ ZHENG WEI $^{1}$, XIANG HU ${ }^{1}$, SHUANGLIN XIANG ${ }^{1}$ and JIAN ZHANG ${ }^{1}$ \\ ${ }^{1}$ Key Laboratory of Protein Chemistry and Developmental Biology of the State Education Ministry of China, \\ College of Life Science, Hunan Normal University; ${ }^{2}$ College of Basic Medical Sciences, Hunan University \\ of Chinese Medicine; ${ }^{3}$ School of Medicine, Hunan Normal University, Changsha, P.R. China
}

Received October 11, 2012; Accepted November 12, 2012

DOI: $10.3892 /$ or.2012.2160

\begin{abstract}
Eps8 was initially identified as a substrate of the epidermal growth factor receptor. Overexpression of Eps8 leads to increased mitogenic signaling and malignant transformation. However, little is known concerning the importance of Eps8 in human gliomas. In this study, we found that Eps8 was overexpressed in $56.6 \%$ of human gliomas (WHO grades III and IV) compared with adjacent normal brain tissues by immunohistochemical analysis. The U251 human glioma cell line stably expressing Eps8 was established by G418 screening, and the ectopic expression of Eps8 enhanced U251 glioma cell growth and survival by cell survival, MTT and liquid colony formation assays. By contrast, the lentiviral expression of Eps8 siRNA in SHG-44 cells resulted in a significant reduction in cellular growth and proliferation. Furthermore, Eps8 modulated the levels of phosphorylated extracellular signal-regulated protein kinase (ERK), phosphorylated serine-threonine protein kinase Akt and $\beta$-catenin expression in glioma cell lines and tissues. These results suggest that Eps 8 is overexpressed in human gliomas, and affects glioma cell growth possibly by regulating ERK and Akt/ $\beta$-catenin signaling. Therefore, Eps8 may represent a novel potential target in human glioma therapy.
\end{abstract}

\section{Introduction}

Gliomas are the most common form of primary human brain tumors, and the incidence rate is approximately $40-50 \%$

Correspondence to: Professor Shuanglin Xiang or Professor Jian Zhang, Key Laboratory of Protein Chemistry and Developmental Biology of the State Education Ministry of China, College of Life Science, Hunan Normal University, Lushan Road No. 14, Hunan, Changsha 410081, P.R. China

E-mail: xshlin@hunnu.edu.cn

E-mail: zhangjian@hunnu.edu.cn

*Contributed equally

Key words: EGF receptor substrate 8, glioma, cellular growth, lentiviral RNAi system, extracellular signal-regulated protein kinase, phosphorylated serine-threonine protein kinase Akt, $\beta$-catenin among all brain tumors. Malignant gliomas are associated with a poorly prognosis and survival rate because of their highly invasive behavior, which results in incomplete tumor resection and a major obstacle to any therapeutic remedies including surgery, radiotherapy and chemotherapy (1). In recent years molecular-targeted therapies are a new strategy for the treatment of gliomas to block cell signaling that governs cell growth, survival and migration (2). Epidermal growth factor receptor (EGFR) gene amplification and overexpression are a striking feature of high-grade gliomas (3). EGFR inhibitors were found to promote significant clinical response in a subset of malignant glioma patients $(4,5)$.

Eps8 is a substrate of both the receptor and non-receptor tyrosine kinases $(6,7)$. Eps8 increased the mitogenic response to EGF and contributes to EGF-dependent cellular tranformation $(6,8,9)$. Eps8, Abi-1 and Sos1 form a tricomplex and induce Rac-specific guanine nucleotide exchange factor (GEF) activity, and enhance Rac-dependent actin remodeling (10-12). Further studies showed that the C-terminal effector region of Eps8 binds with F-actin and localizes to main sites of actin remodeling, such as lamellipodia, filopodia and membrane ruffles $(10,11)$. Recently, many studies have shown that Eps8 functions in various solid tumors, including squamous cell carcinoma, pancreatic cancer and cervical cancer, as well as colon cancer and pituitary tumors (13-18). Moreover, high expression and concomitant tyrosine phosphorylation of Eps8 were detected in tumor cell lines (9). Eps8 increases cell growth and motility, by the upregulation of FOXM1, FAK, MMP-9, ERK, Akt, cyclins D1, D3 and E, and the downregulation of P53 and p21Waf1/Cip1 in cancer cells $(13-15,17,18)$. Additionally, the Sp1 inhibitor mithramycin downregulates Eps8 expression and inhibits human epithelial carcinoma cell proliferation and migration, which clearly suggests the Eps8 knockdown may exert an anticancer ability (19).

Although recent studies imply a critical role for Eps8 in the development of many malignancies, the expression and function of Eps8 in gliomas have not been fully elucidated. In this study, we attempted to investigate the expression of Eps8 in glioma tissues and cell lines, as well as the role of Eps8 in glioma malignancy, including cell proliferation and survival. Overexpression of Eps8 significantly enhanced the growth of the glioma cells, whereas inhibition of Eps8 by a lentiviral RNAi system had an 
opposite effect. Our results demonstrated that Eps8 serves as a novel growth regulator in glioma cells and could be a novel target for the diagnosis and treatment of gliomas.

\section{Materials and methods}

Immunohistochemistry. A total of 53 brain tumors including 10 WHO grade III and 43 WHO grade IV gliomas was examined. Patient characteristics are listed in Table I. Seven adjacent normal human brain tissues were used as the control. The study was approved by the Hunan Normal University Human Ethics Committee, and informed consent was obtained from all patients. The immunohistochemistry was analyzed on formalin-fixed and paraffin-embedded samples. Endogenous peroxidase was blocked with $3 \% \mathrm{H}_{2} \mathrm{O}_{2}$ in methanol for $10 \mathrm{~min}$. The mouse monoclonal anti-Eps8 antibody (BD Biosciences, CA, USA) at a 1:500 dilution or mouse control IgG was added at $4^{\circ} \mathrm{C}$ overnight. The sections were incubated with HRP-conjugated goat anti-mouse secondary antibody (SigmaAldrich Corp., St. Louis, MO, USA) for $30 \mathrm{~min}$ and then with 3,3-diaminobenzidine (DAB) $/ \mathrm{H}_{2} \mathrm{O}_{2}$ for $5 \mathrm{~min}$. The sections were counterstained with hematoxylin, mounted and photographed using an optical microscope. The percentage of tumor cells stained was scored as: 0 (no cell staining), + or $1(\leq 30 \%)$, ++ or 2 (31-60\%), and +++ or 3 (61-100\%). Some samples with a staining between two score values were given 0.5.

Cell culture. The human neuroblastoma cell line SH-SY5Y was cultured in a 1:1 mixture of F12 and Eagle's minimum essential medium (EMEM) supplemented with non-essential amino acids, glutamine, sodium pyruvate, penicillin, streptomycin, and 10\% fetal bovine serum (FBS). HEK293FT and 293T cells, as well as human glioma A172, U251, and SHG-44 cells were cultured in complete Dulbecco's modified Eagle's medium (DMEM) containing penicillin, streptomycin and $10 \%$ fetal bovine serum. Cells were maintained in a humidified atmosphere of $5 \% \mathrm{CO}_{2}$ and $95 \%$ air at $37^{\circ} \mathrm{C}$.

Plasmid constructs and establishment of U251 cell lines that express GFP and GFP-Eps8. To generate the pEGFP-C3-Eps8 plasmid, full-length human Eps8 was released by EcoRI and XhoI digestion of a Myc-tagged human Eps8 expression vector pCMV-Myc-Eps8 (20) and subcloned into the EcoRI and SalI sites of vector pEGFP-C3. U251 cells were transfected with pEGFP-C3-Eps8 or pEGFP-C3 control plasmids using Lipofectamine 2000 (Invitrogen, Carlsbad, CA, USA). Stably transfected cells were isolated by G418 screening $(21,22)$. The transfection efficiency was detected using a fluorescence microscope (Zeiss Axioskop 2, LLC, USA).

Western blotting. Samples were denatured in sample buffer [2\% sodium dodecyl sulfate (SDS), $10 \%$ glycerol, $2.5 \%$ $\beta$-mercaptoethanol, and bromophenyl blue] and heated to $100^{\circ} \mathrm{C}$ for $5 \mathrm{~min}$ before gel electrophoresis. Samples were then separated on a $10 \%$ SDS-PAGE gel and transferred to a polyvinylidene difluoride (PVDF) membrane. The blots were probed with rabbit polyclonal anti-GFP antibody, mouse monoclonal antibodies against $\beta$-catenin, $\beta$-actin, Akt, phosphorylated Akt, ERK and phosphorylated ERK (Santa Cruz Biotechnology, Santa Cruz, CA, USA). HRP-conjugated goat anti-rabbit and
Table I. Patient characteristics.

No. of patients $(\%)$

Total number

Age, range in years (average)

$30-65(75)$

Gender

Male

Female

Grade of glioma

III

IV

Normal tissue 7

goat anti-mouse secondary antibodies (Sigma) were used for detection. The signal was visualized with SuperSignal West Femto Maximum Sensitivity Substrate (Pierce Biotechnology, Rockford, IL, USA).

Eps8 RNAi lentivirus generation. Four siRNA sequences targeting Eps8 (NM_004447.5) were from the positions (siRNA1, 93-111; siRNA2, 246-264; siRNA3, 678-696; siRNA4, 1974-1992) relative to the start codon and synthesized by Shanghai Integrated Biotech Solutions Co. Ltd., China. After testing knockdown efficiencies, stem-loop DNA oligonucleotides were synthesized by Shanghai GeneChem Co. Ltd. (shRNA1 sense, 5'-CCG GAC GGA CAG AGA ACA TGG TTC ACT CGA GTG AAC CAT GTT CTC TGT CCG TTT TTT G-3'; antisense, 5'-AAT TCA AAA AAC GGA CAG AGA ACA TGG TTC ACT CGA GTG AAC CAT GTT CTC TGT CCG T-3'; shRNA2 sense, 5'-CCG GAC CAC TGT TGA TGA TGG AAT ACT CGA G TA TTC CAT CAT CAA CAG TGG TTT TTG-3'; antisense, 5'-AAT TCA AAA AAC CAC TGT TGA TGA TGG AAT ACT CGA GTA TTC CAT CAT CAA CAG TGG T-3') and cloned into the lentivirus-based RNAi vector pGCSIL-GFP. A nontargeting stem-loop DNA was also inserted into the pGCSIL-GFP vector as a negative control (NC). Lentiviral particles were prepared as described previously (23). SHG-44 cells were infected with Eps8-RNAilentivirus or NC-GFP-lentivirus and detected on day 6 by a fluorescence microscope and western blot analysis.

Cell proliferation assays. For cell survival assays, 5,000 cells stably expressing GFP-Eps8 or GFP and parental U251 cells were plated in triplicate in 6-well plates in complete medium containing $400 \mu \mathrm{g} / \mathrm{ml} \mathrm{G} 418$. For SHG-44 cells, 5,000 cells expressing Eps8-RNAi-LV or NC-GFP-LV were plated in triplicate in 6-well plates. After 8-10 days, cell numbers were counted with a hemocytometer after trypan blue staining of viable cells.

For the cell viability assay, 3,000 cells were seeded in octuplicate in 96-well plates. On days 1, 4, 8 and 10 or days $1,3,5$ and 8 , cells were treated with $1 \mathrm{mg} / \mathrm{ml} \mathrm{3-(4,5-dimethylthiazol-}$ 2-yl)-2,5-diphenytetrazolium bromide (MTT) at $37^{\circ} \mathrm{C}$ for $4 \mathrm{~h}$. Then $100 \mu 1$ dimethylsulfoxide (DMSO) was added to the medium to dissolve the formazan crystals. The absorbency 


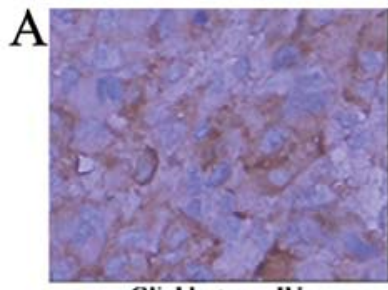

Glioblastoma IV $++$

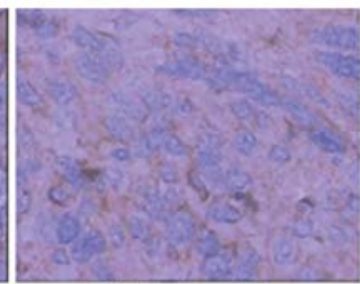

Glioblastoma IV $++$

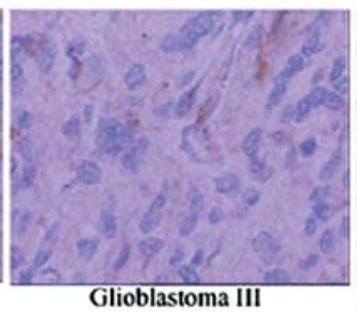

$+/ 0$

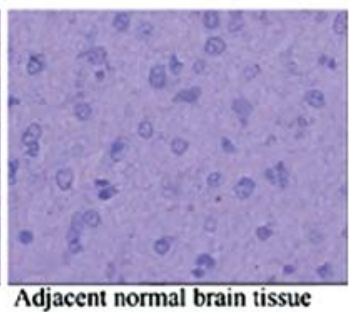

$-$
B

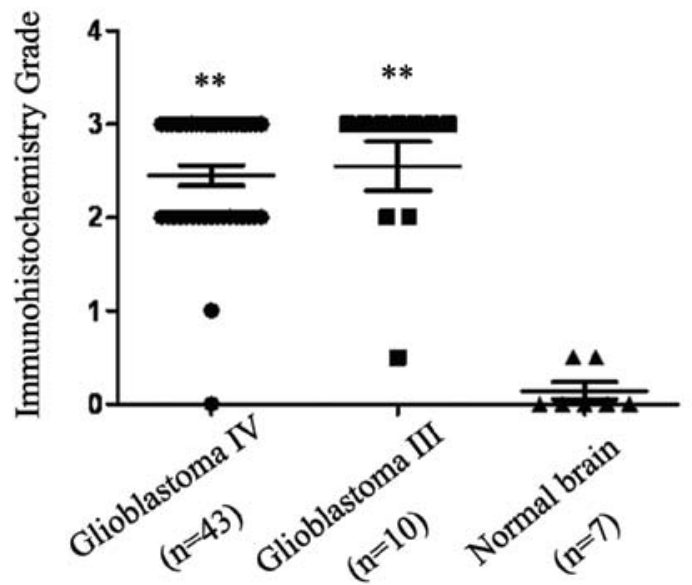

$\mathrm{C}$

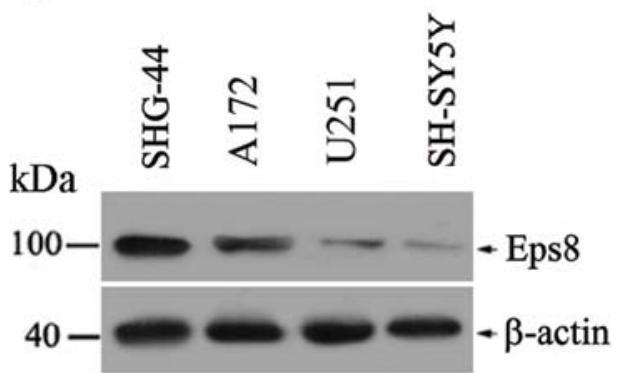

Ratio:1.18 $0.56 \quad 0.130 .06$

Figure 1. Eps8 expression in glioma tissues and cell lines. (A) Immunohistochemical analysis of Eps8 expression in 53 brain tumors and normal brain tissues Positive Eps8 staining (brown) was shown in the cytoplasm of tumor cells whereas the nucleus was stained blue with hematoxylin. (B) Immunohistochemical grades of gliomas and normal tissues stained with mouse monoclonal anti-Eps8. Immunohistochemical staining intensity for each sample was scored for grades 0-3. Each symbol represents a sample from an individual patient. Statistical comparisons between gliomas and normal tissues were carried out according to the Student's t-test. ${ }^{* *}$ p $<0.001$. (C) Western blot analysis of Eps8 protein levels in glioma cell lines. $\beta$-actin was used as a loading control. Densitometric analysis of Eps $8 / \beta$-actin ratio was performed with Gel-Pro Analyzer 4.0 software.

value at $490 \mathrm{~nm}$ in each well was obtained using a spectrophotometer (UV-2102C, Changsha, China).

The liquid colony formation was carried out. 1,000 cells were seeded in triplicate in 6-well plates, and cells were grown for 2 weeks in a humidified incubator at $37^{\circ} \mathrm{C}$ with $5 \% \mathrm{CO}_{2}$. Colonies were fixed with methanol, stained with Giemsa (BBI International, Cardiff, UK) and photographed with a digital camera (Olympus, Tokyo, Japan). Only colonies containing over 30 cells were counted.

Statistical analysis. Data are presented as the means \pm SD from at least three independent experiments. The significance of difference was assessed using the Student's t-test. Values of $\mathrm{p}<0.05$ were considered statistically significant.

\section{Results}

Expression of Eps8 in glioma tissues and cell lines. The expression level of Eps8 was examined in 10 WHO grade III, 43 WHO grade IV gliomas and 7 adjacent normal human brain tissues by immunohistochemical analysis using mouse monoclonal Eps8-specific antibody. We found that Eps8 was completely localized in the cytoplasm (Fig. 1A). Eps8 expression was detected in $30(56.6 \%)$ of the 53 brain tumors with strong staining (3+), $20(37.7 \%)$ of the 53 gliomas were moderately stained (2+), and $3(5.7 \%)$ were weakly stained or negative for Eps8 expression (+/0), which showed Eps8 was significantly highly expressed in the gliomas $(\mathrm{P}<0.001$; Student's t-test). A complete loss of Eps8 expression was observed in normal brain tissues (Fig. 1B). Therefore, Eps8 expression was markedly increased in the human glioma tissues when compared with expression in the normal brain tissues.

We next analyzed the expression of Eps8 protein in three human glioma cell lines (A172, U251 and SHG-44) and human neuroblastoma cell line SH-SY5Y. Higher expression of Eps8 protein was evident in the SHG-44 cells when compared with that in the A172 and U251 cells. SH-SY5Y cells demonstrated a slight level of Eps8 protein (Fig. 1C). Thus, the level of Eps8 expression differed in the two glioma cell lines. U251 and SHG-44 cell lines were further selected to overexpress and knockdown Eps8 protein, respectively.

Eps8 overexpression enhances the proliferation of the U251 human glioma cell line. To further investigate the role of Eps8 in human glioma cells, we constructed the pEGFP-C3/Eps8 plasmid and established U251 cell lines stably overexpressing GFP/Eps8 and GFP by G418 screening (Fig. 2A). The level of Eps8 overexpression was demonstrated by western blotting. As shown in Fig. 2B, U251-GFP and U251-GFP/Eps8 cells were found to express the target proteins at high levels.

We next examined whether Eps8 overexpression in U251 glioma cells promotes cell proliferation. Cells were plated in triplicate in 6-well plates, and the cell number was counted on day 10. We found that the overexpression of Eps8 in U251 cells 


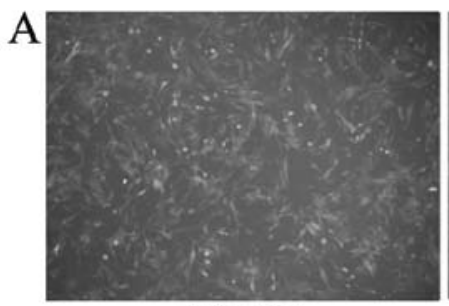

U251-GFP

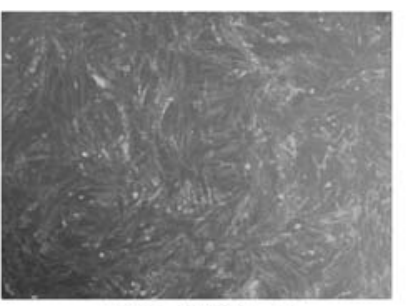

U251-GFP/Eps8

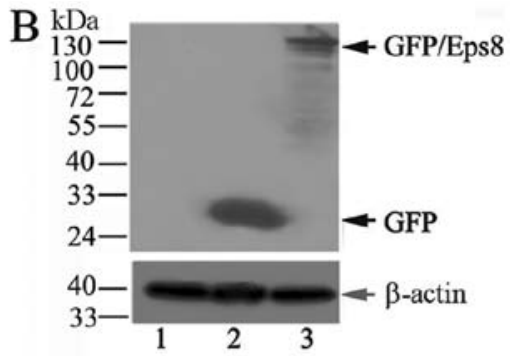

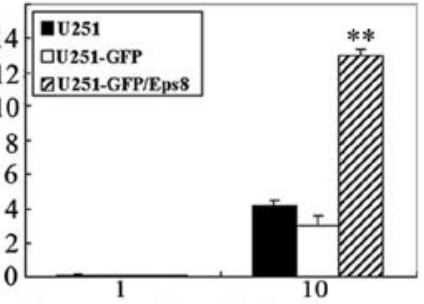

Time (day)

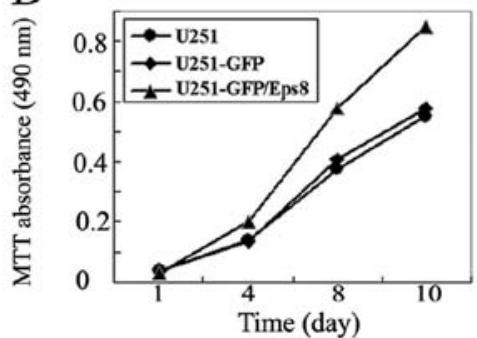

$\mathrm{E}$
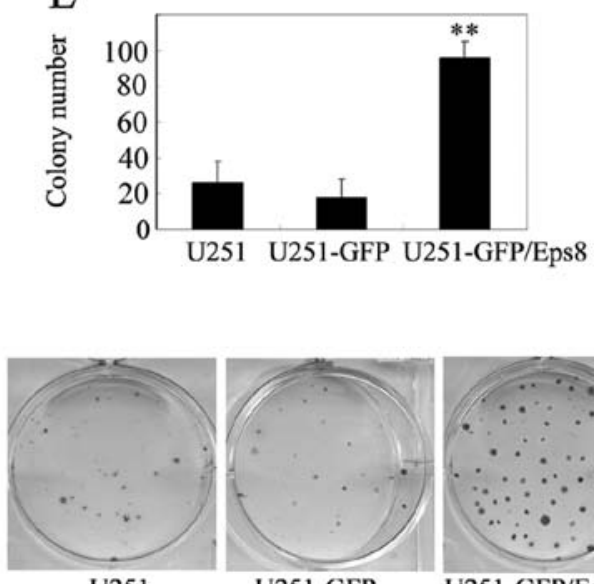

$\mathrm{U} 251$

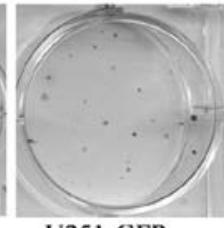

U251-GFP

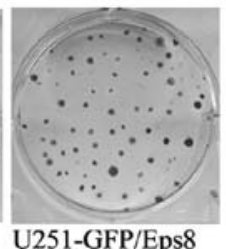

Figure 2. Effects of Eps8 overexpression on U251 glioma cell survival and proliferation. (A) Immunofluorescence assay confirming the establishment of U251 cells stably expressing GFP/Eps8 and GFP. (B) Western blot assay of GFP and GFP/Eps8 expression in U251 cells using anti-GFP antibodies. $\beta$-actin was served as a loading control. Lane 1, U251 cells; lane 2, GFP-transfected U251 cells; lane 3, GFP/Eps8-transfected U251 cells. (C) Cell survival assay of GFP/ Eps8-transfected U251 cells, GFP-transfected U251 cells and parental U251 cells. Cells $(5,000)$ were plated into 6-well plates in triplicate under the same condition, grown in RPMI-1640 with 10\% FBS for 10 days and stained with trypan blue in PBS, and viable cells were counted. (D) MTT assays of mock or transfected U251 cells. Cells $(3,000)$ were plated in octuplicate in 96-well plates and grown in RPMI-1640 with 10\% FBS. The absorbance was analyzed following 1, 4, 8 and 10 days. (E) Liquid colony formation analysis of mock or transfected U251 cells. Cells $(1,000)$ were seeded in triplicate in 6-well plates, and grown for 2 weeks. Colonies were fixed with methanol, stained with Giemsa, images (lower panel) were captured and the number of colonies (upper panel) were counted. These data represent at least three independent experiments with similar results. ${ }^{* *} \mathrm{p}<0.01$, compared with parental and control cells.
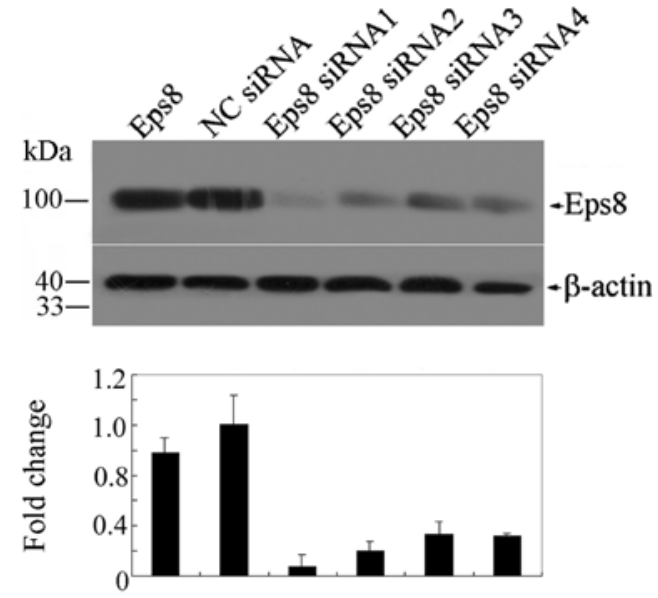

Figure 3. Identification of Eps8 siRNAs. HEK293FT cells were transfected with pCMV-Myc-Eps8 and Eps8 siRNAs, the Eps8 knockdown efficiency was detected by western blotting. $\beta$-actin was used as a loading control. Densitometric analysis of Eps8/ $\beta$-actin ratio was performed with Gel-Pro Analyzer 4.0 software.

resulted in an increased cellular growth compared with the controls (Fig. 2C). Similarly, we tested the cell viability using
MTT assay, Eps8 overexpression resulted in a striking increase in the number of viable cells (Fig. 2D). Furthermore, the liquid colony formation assay demonstrated a great increase in the numbers of colonies of the Eps8-overexpressing U251 cells (Fig. 2E). Taken together, these data suggest that Eps8 contributes to glioma cell survival and proliferation in vitro.

Eps8 knockdown antagonizes the proliferation of the SHG-44 human glioma cell line. The above results indicate that Eps8 significantly promotes U251 cellular proliferation. To gain further supporting evidence, we knocked down Eps8 expression using shRNA and determined whether shRNA-mediated Eps8 knockdown inhibits the proliferative effects. Four siRNA sequences targeting different regions of Eps8 mRNA were constructed and their abilities to knock down Eps8 expression in transfected HEK293FT cells were demonstrated by western blotting. As shown in Fig. 3, the four sequences knocked down the Eps8 gene to different levels, especially siRNA1 and siRNA2.

Consequently, siRNA1 and siRNA2 were designed to construct the lentivirus-based RNAi vector pGCSIL-Eps8. Packaging plasmids and pGCSIL-Eps8 were cotransfected into the $293 \mathrm{~T}$ cells. The fluorescence intensity was markedly 


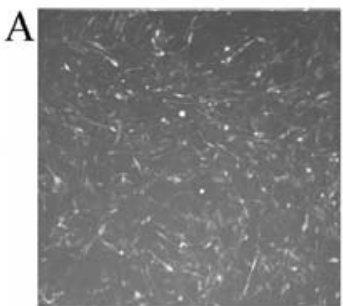

SHG-44-NC-GFP-LV

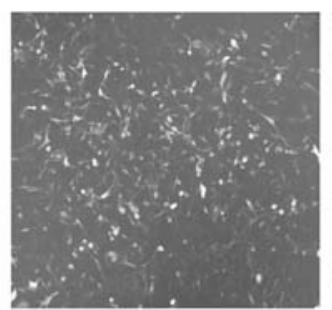

SHG-44-Eps8-RNAi 1-LV

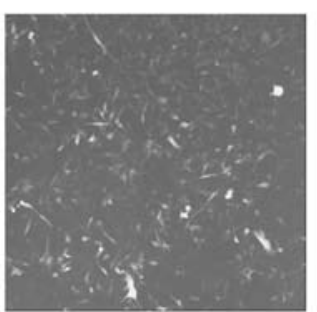

SHG-44-Eps8-RNAi 2-LV

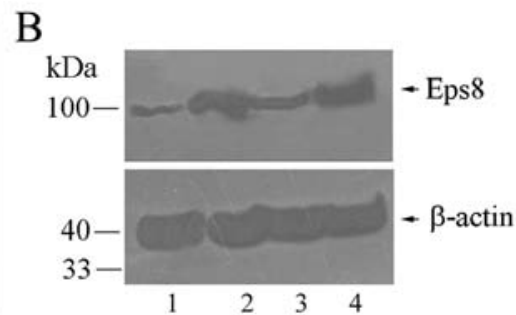

$\mathrm{C}$
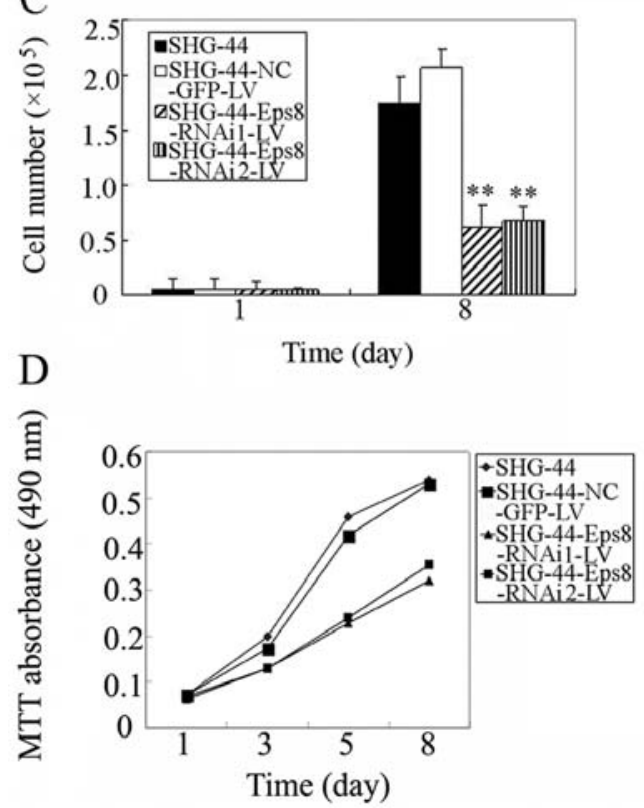

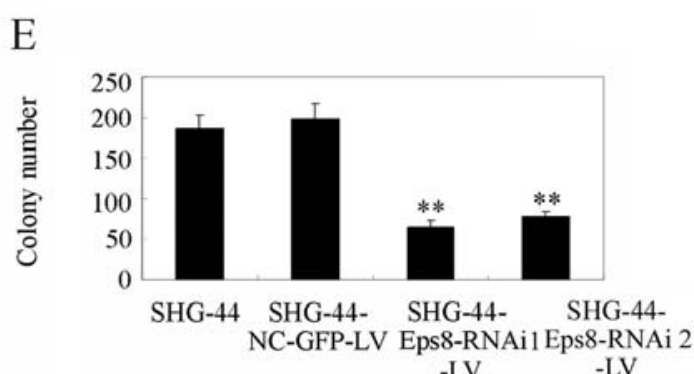

$-\mathrm{LV}$

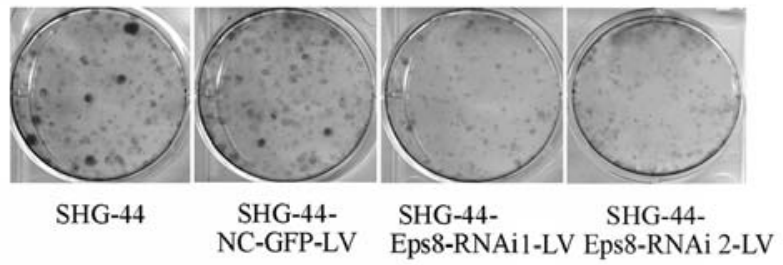

Figure 4. Effects of Eps8 knockdown on SHG-44 glioma cell survival and proliferation. (A) Immunofluorescence assays for the measurement of the transduction efficiency of SHG-44 cells infected with shRNA1 or shRNA2. (B) The Eps8 knockdown efficiency was detected by western blot analysis with mouse monoclonal anti-Eps8 antibody. $\beta$-actin was used as a loading control for total lysate samples. Lane 1, Eps8 shRNA 1-transfected SHG-44 cells; lane 2 , SHG-44 cells; lane 3, Eps8 shRNA2-transfected SHG-44 cells; lane 4, GFP NC-transfected SHG-44 cells. (C) Cell survival assay of Eps8 shRNA-transfected SHG-44 cells, GFP NC-transfected SHG-44 cells and parental SHG-44 cells as described in Materials and methods. (D) MTT assays of mock or transfected SHG-44 cells. SHG-44 cells (3,000) were plated and grown, and the absorbance was analyzed on days $1,3,5$ and 8 . (E) Liquid colony formation analysis of mock or transfected SHG-44 cells. SHG-44 cells $(2,000)$ were seeded and grown for 2 weeks. Colonies were fixed with methanol, stained with Giemsa, images (lower panel) were captured and the number of colonies were counted (upper panel). These data represent at least three independent experiments with similar results. ${ }^{* *}$ p $<0.01$, compared with parental and control cells.

increased 4 days after transfection and the transfection efficiency was nearly $90 \%$ in the SHG-44 cells (Fig. 4A). Meanwhile, the expression of Eps8 proteins was detected by western blot analysis. Eps8 shRNA1 and shRNA2 significantly suppressed the expression of Eps8 protein when compared with the negative control shRNA (Fig. 4B).

Given that Eps8 is a critical regulator of glioma cell proliferation, we examined the effect of Eps8 on SHG-44 glioma cell proliferation. As shown in Figs. 4C and D, Eps8 knockdown markedly inhibited the proliferation of SHG-44 cells, whereas control shRNA exhibited no effect. The colony formation assays showed that deletion of Eps 8 expression produced a significant decrease in the clonogenicity of SHG-44 cells, while control shRNA produced no effect (Fig. 4E). These data clearly indicate that Eps8 knockdown reduces glioma cell proliferation.

Eps8 affects the expression of phosphorylated ERK, Akt and $\beta$-catenin in glioma cell lines and tissues. Since Eps8 regulates many genes involved in the development of human cancers, we determined to investigate whether Eps8 influences these target genes in glioma cell lines and tissues. As shown in Fig. 5A, Eps8 overexpression had no effect on ERK and Akt protein levels, while it increased the levels of phosphorylated ERK and Akt expression. Moreover, the level of $\beta$-catenin was concomitantly enhanced in Eps8-transfected cells. Likely, Eps8 knockdown decreased the expression of phosphorylated ERK, phosphorylated Akt and $\beta$-catenin, but not ERK or Akt (Fig. 5B).

We then analyzed the expression of Eps8 and target genes in normal brain tissues and gliomas. Eps8 had a significantly higher expression in high grade gliomas (WHO III-IV) than in low grade gliomas (WHO I-II) and normal brain tissues. In addition, the expression of Eps8 revealed broadly paralleled expression of target genes in the gliomas and normal brain tissues, particularly phosphorylated ERK and phosphorylated Akt. However, the levels of $\beta$-catenin were partially correlated with Eps8 expression in certain samples (Fig. 5C). Taken together, these data show that Eps8 regulates cell proliferation and survival, at least in part, by affecting phosphorylated ERK and $\mathrm{Akt} / \beta$-catenin activities. 


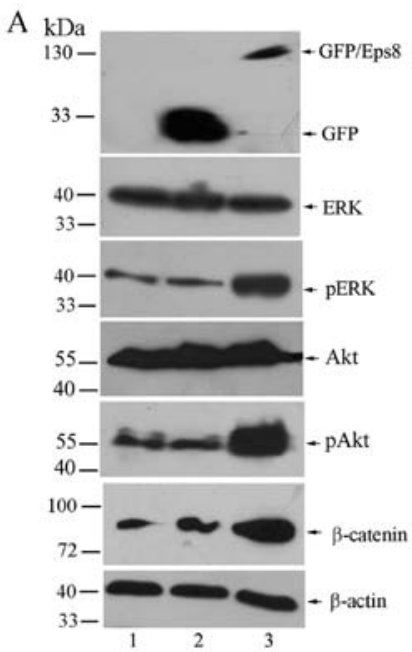

$\mathrm{B} \mathrm{kDa}$
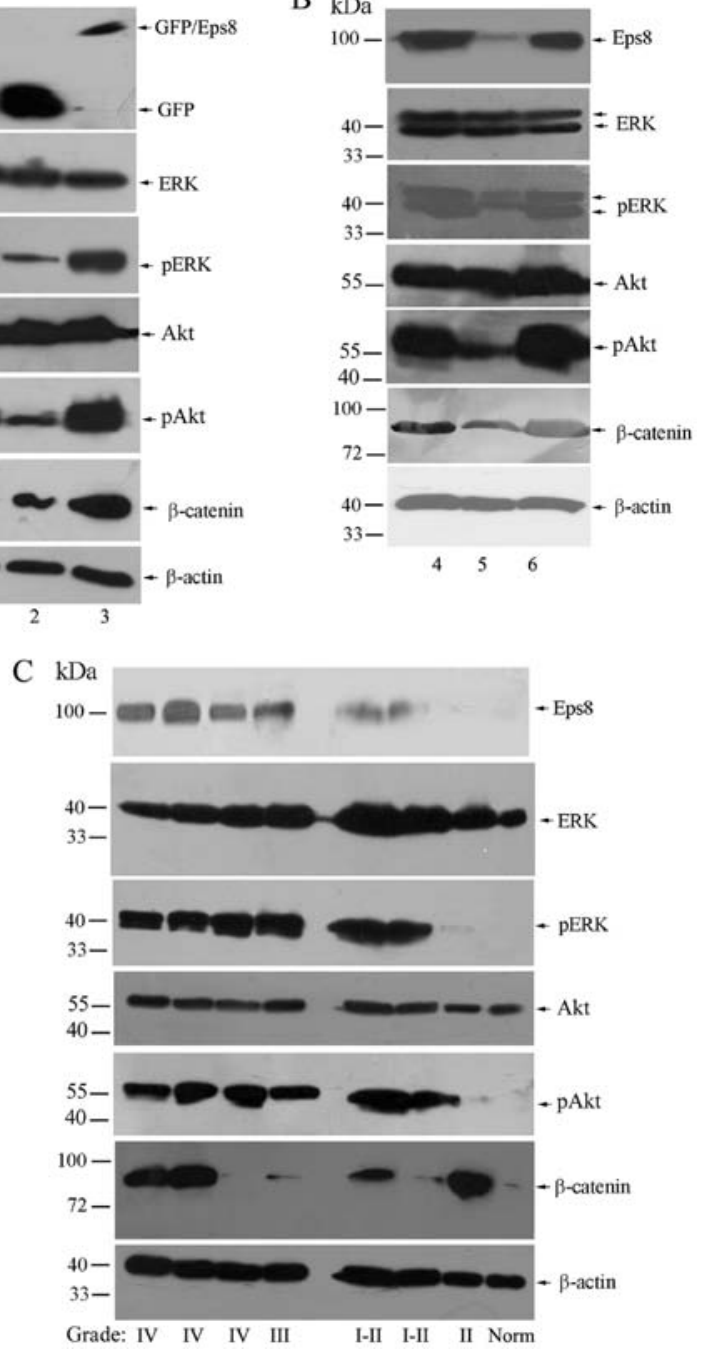

Figure 5. Eps8 modulates the expression of phosphorylated ERK, Akt and $\beta$-catenin in glioma cells and tissues. (A and B) Western blotting was carried out to detect the effect of Eps8 overexpression and knockdown on the expression of target genes ERK, Akt and $\beta$-catenin. Lane 1, U251 cells; lane 2, GFP-transfected U251 cells; lane 3, GFP/Eps8-transfected U251 cells; lane 4 , SHG-44 cells; lane 5, Eps8 shRNA1-transfected SHG-44 cells; lane 6, GFP NC-transfected SHG-44 cells. (C) Western blotting was performed to detect the relationship of Eps8 and target genes ERK, Akt and $\beta$-catenin in glioma samples. $\beta$-actin was used as a loading control for total lysate samples.

\section{Discussion}

The human Eps8 family includes Eps8 and three related proteins Eps8L1, Eps8L2, and Eps8L3. Eps8Ls display 27-42\% identity to Eps8. However, Eps8L2 and Eps8 display the highest degree of amino acid identity within the family, which explains for the lack of overt phenotype in Eps8 null mice. Only Eps8 is specifically expressed in the brain of the 16.5 dpc mouse embryo among the Eps8 family (24). In addition, it has also been reported that Eps8 is enriched in the growth cone and filopodia of developing hippocampal neurons (25). Thus, Eps8 is likely to function in the process of neurodevelopment.

Previous data showed that Eps8 is important in regulating the progression of certain types of human cancers. However, the role of Eps8 in human gliomas has not been previously reported. In the present study, we provide evidence concerning the expression of Eps8 in glioma tissues and cell lines. As assessed by immunohistochemical analysis, Eps8 expression was detectable in the majority of the glioma specimens examined. More importantly, Eps 8 protein was significantly higher in $56.6 \%$ of high grade (III and IV) glioma tissues compared to normal brain tissues. Accordingly, 3 glioma cell lines were used to demonstrate that the protein level of Eps8 was upregulated in glioma specimens, which was consistent with previous evidence that high expression of Eps8 is noted in tumor cell lines and tissues (9). Thus, Eps8 expression may clinically designate high grade malignant gliomas.

The evidence presented here also verified the effect of Eps8 on glioma cell proliferation. We established a stable cell line overexpressing Eps8 using U251 cells which have low constitutive expression of Eps8. We used this cell line to investigate the effect of Eps8 overexpression on glioma cellular growth in vitro. Eps8 overexpression markedly enhanced cell proliferation. Conversely, we constructed lentivirus-based RNAi vector pGCSIL-Eps8 to knock down Eps8 expression in the SHG-44 cell line, which exhibits high expression of Eps8. Depletion of Eps8 reversed the growth phenotype of Eps8-overexpressing glioma cells. Our data strongly indicate that Eps8 expression is critical for the cell proliferation of malignant gliomas and is possibly involved in malignant progression in human gliomas.

Eps8, Abi-1 and Sos1 are known to form a tricomplex and induce Rac-specific guanine nucleotide exchange factor (GEF) activity, transduce signals from Ras to Rac and enhance Rac-dependent actin remodeling (10-12). Additional, Eps8 activates Ras and Raf. Raf then activates MEK, which in turn activates ERK (26). ERK is known to be a critical regulator that mainly participates in the stimulation of cell proliferation and survival (27). We demonstrated that Eps8 plays an important role in the growth of glioma cells by regulating the expression of phosphorylated ERK.

$\beta$-catenin functions as a core member of canonical Wnt signaling. When Wnt binds to its receptor, transcription factor $\beta$-catenin accumulates in the cytoplasm and then translocates to the nucleus where it subsequently transactivates Wnt pathway downstream targets known to be crucial in cancer develoment $(28,29)$. $\beta$-catenin is an oncogene in a broad range of human cancers $(29,30)$. For example, overexpression of $\beta$-catenin contributes to glioma progression $(31,32)$. Eps8 was reported to trigger increased proliferation through activation of PI3K via Akt $(16,33)$. Moreover, $\beta$-catenin can be transactivated via the PI3K/Akt pathway $(34,35)$. Therefore, our finding that Eps8 knockdown in glioma cells downregulates the overall level of phosphorylated Akt and $\beta$-catenin and vice versa, suggesting that Eps8 may effectively regulate Akt/ $\beta$-catenin signaling in gliomas.

In summary, this study reveals the biological significance of Eps8 expression in gliomas. We report that Eps8 is highly expressed in malignant gliomas, which correlates with glioma cell proliferation. We found that Eps8 mediates cell proliferation and survival of glioma cells, at least in part, by affecting phosphorylated ERK and Akt/ $\beta$-catenin activities. These data suggest that Eps8 may be a potential therapeutic target against human gliomas. 


\section{Acknowledgements}

This study was supported by the National Natural Science Foundation of China (nos. 30900827, 81071656, 81071696, 81172112), the Opening Fund of the Key Laboratory of Protein Chemistry and Developmental Biology of the Ministry of Education, Hunan Normal University.

\section{References}

1. Giese A, Bjerkvig R, Berens ME and Westphal M: Cost of migration: invasion of malignant gliomas and implications for treatment. J Clin Oncol 21: 1624-1636, 2003.

2. Liu TJ, LaFortune T, Honda T, Ohmori O, Hatakeyama S, Meye T, Jackson D, de Groot J and Yung WK: Inhibition of both focal adhesion kinase and insulin-like growth factor-I receptor kinase suppresses glioma proliferation in vitro and in vivo. Mol Cancer Ther 6: 1357-1367, 2007.

3. Ekstrand AJ, James CD, Cavenee WK, Seliger B, Pettersson RF and Collins VP: Genes for epidermal growth factor receptor, transforming growth factor alpha, and epidermal growth factor and their expression in human gliomas in vivo. Cancer Res 51: 2164-2172, 1991.

4. Nakamura JL: The epidermal growth factor receptor in malignant gliomas: pathogenesis and therapeutic implications. Expert Opin Ther Targets 11: 463-472, 2007.

5. Mellinghoff IK, Wang MY, Vivanco I, Haas-Kogan DA, Zhu S, Dia EQ, Lu KV, Yoshimoto K, Huang JH, Chute DJ, Riggs BL, Horvath S, Liau LM, Cavenee WK, Rao PN, Beroukhim R, Peck TC, Lee JC, Sellers WR, Stokoe D, Prados M, Cloughesy TF, Sawyers CL and Mischel PS: Molecular determinants of the response of glioblastomas to EGFR kinase inhibitors. N Engl J Med 353: 2012-2024, 2005.

6. Fazioli F, Minichiello L, Matoska V, Castagnino P, Miki T, Wong WT and Di Fiore PP: Eps8, a substrate for the epidermal growth factor receptor kinase, enhances EGF-dependent mitogenic signals. EMBO J 12: 3799-3808, 1993.

7. Maa MC, Lai JR, Lin RW and Leu TH: Enhancement of tyrosyl phosphorylation and protein expression of eps 8 by v-Src. Biochim Biophys Acta 1450: 341-351, 1999.

8. Castagnino P, Biesova Z, Wong WT, Fazioli F, Gill GN and Di Fiore PP: Direct binding of eps 8 to the juxtamembrane domain of EGFR is phosphotyrosine- and SH2-independent. Oncogene 10: 723-729, 1995

9. Matoskova B, Wong WT, Salcini AE, Pelicci PG and Di Fiore PP: Constitutive phosphorylation of eps8 in tumor cell lines: relevance to malignant transformation. Mol Cell Biol 15: 3805-3812, 1995.

10. Scita G, Nordstrom J, Carbone R, Tenca P, Giardina G, Gutkind S, Bjarnegard M, Betsholtz C and Di Fiore PP: Eps8 and E3B1 transduce signals from Ras to Rac. Nature 401: 290-293, 1999.

11. Scita G, Tenca P, Areces LB, Tocchetti A, Frittoli E, Giardina G, Ponzanelli I, Sini P, Innocenti M and Di Fiore PP: An effector region in Eps8 is responsible for the activation of the Rac-specific GEF activity of Sos-1 and for the proper localization of the Rac-based actin-polymerizing machine. J Cell Biol 154: 1031-1044, 2001.

12. Chen H, Wu X, Pan ZK and Huang S: Integrity of SOS1/EPS8/ ABI1 tri-complex determines ovarian cancer metastasis. Cancer Res 70: 9979-9990, 2010

13. Wang H, Teh MT, Ji Y, Patel V, Firouzabadian S, Patel AA, Gutkind JS and Yeudall WA: EPS8 upregulates FOXM1 expression, enhancing cell growth and motility. Carcinogenesis 31 1132-1141, 2010

14. Chen YJ, Shen MR, Chen YJ, Maa MC and Leu TH: Eps8 decreases chemosensitivity and affects survival of cervical cancer patients. Mol Cancer Ther 7: 1376-1385, 2008.

15. Maa MC, Lee JC, Chen YJ, Chen YJ, Lee YC, Wang ST, Huang CC, Chow NH and Leu TH: Eps8 facilitates cellular growth and motility of colon cancer cells by increasing the expression and activity of focal adhesion kinase. J Biol Chem 282: 19399-19409, 2007.

16. Wang H, Patel V, Miyazaki H, Gutkind JS and Yeudall WA: Role for EPS8 in squamous carcinogenesis. Carcinogenesis 30: 165-174, 2009.
17. Zhang W, Wang L, Liu Y, Xu J, Zhu G, Cang H, Li X, Bartlam M, Hensley K, Li G, Rao Z and Zhang XC: Structure of human lanthionine synthetase C-like protein 1 and its interaction with Eps8 and glutathione. Genes Dev 23: 1387-1392, 2009.

18. Welsch T, Endlich K, Giese T, Buchler MW and Schmidt J: Eps8 is increased in pancreatic cancer and required for dynamic actinbased cell protrusions and intercellular cytoskeletal organization. Cancer Lett 255: 205-218, 2007.

19. Yang TP, Chiou HL, Maa MC and Wang CJ: Mithramycin inhibits human epithelial carcinoma cell proliferation and migration involving downregulation of Eps8 expression. Chem Biol Interact 183: 181-186, 2010.

20. Luo C, Ding XF, Sun YB and Han M: Subcellular location of EPS8 by its expression and preparation of antiserum. J Nat Sci Hunan Normal University 31: 100-104, 2008

21. Mangravite LM, Lipschutz JH, Mostov KE and Giacomini KM: Localization of GFP-tagged concentrative nucleoside transporters in a renal polarized epithelial cell line. Am J Physiol Renal Physiol 280: F879-F885, 2001

22. Schmid JA, Birbach A, Hofer-Warbinek R, Pengg M, Burner U, Furtmuller PG, Binder BR and de Martin R: Dynamics of NF kappa B and Ikappa Balpha studied with green fluorescent protein (GFP) fusion proteins. Investigation of GFP-p65 binding to $\mathrm{DNa}$ by fluorescence resonance energy transfer. J Biol Chem 275: 17035-17042, 2000.

23. Lois C, Hong EJ, Pease S, Brown EJ and Baltimore D: Germline transmission and tissue-specific expression of transgenes delivered by lentiviral vectors. Science 295: 868-872, 2002.

24. Offenhauser N, Borgonovo A, Disanza A, Romano P, Ponzanelli I, Iannolo G, Di Fiore PP and Scita G: The eps8 family of proteins links growth factor stimulation to actin reorganization generating functional redundancy in the Ras/Rac pathway. Mol Biol Cell 15: 91-98, 2004.

25. Menna E, Disanza A,CagnoliC,Schenk U, Gelsomino G,FrittoliE, Hertzog M, Offenhauser N, Sawallisch C, Kreienkamp HJ, Gertler FB, Di Fiore PP, Scita G and Matteoli M: Eps8 regulates axonal filopodia in hippocampal neurons in response to brainderived neurotrophic factor (BDNF). PLoS Biol 7: e1000138, 2009.

26. Hecquet C, Lefevre G, Valtink M, Engelmann K and Mascarelli F: Activation and role of MAP kinase-dependent pathways in retinal pigment epithelial cells: ERK and RPE cell proliferation. Invest Ophthalmol Vis Sci 43: 3091-3098, 2002.

27. Wortzel I and Seger R: The ERK cascade: distinct functions within various subcellular organelles. Genes Cancer 2: 195-209, 2011.

28. Uthoff SM, Eichenberger MR, McAuliffe TL, Hamilton CJ and Galandiuk S: Wingless-type frizzled protein receptor signaling and its putative role in human colon cancer. Mol Carcinog 31: 56-62, 2001.

29. Barker $\mathrm{N}$ and Clevers $\mathrm{H}$ : Catenins, Wnt signaling and cancer. Bioessays 22: 961-965, 2000

30. Polakis P: Wnt signaling and cancer. Genes Dev 14: 1837-1851, 2000.

31. Liu X WL, Zhao S, Ji X, Luo Y and Ling F: $\beta$-Catenin overexpression in malignant glioma and its role in proliferation and apoptosis in glioblastoma cells. Med Oncol 28: 608-614, 2011.

32. Zhang J HK, Shi Z, Zou J, Wang Y, Jia Z, Zhang A, Han L, Yue X, Liu N, Jiang T, You Y, Pu P and Kang C: High $\beta$-catenin/ Tcf-4 activity confers glioma progression via direct regulation of AKT2 gene expression. Neuro Oncol 13: 600-609, 2011.

33. Xu M, Shorts-Cary L, Knox AJ, Kleinsmidt-DeMasters B, Lillehei K and Wierman ME: Epidermal growth factor receptor pathway substrate 8 is overexpressed in human pituitary tumors: role in proliferation and survival. Endocrinology 150: 2064-2071, 2009.

34. Kanneganti M, Mino-Kenudson M and Mizoguchi E: Animal models of colitis-associated carcinogenesis. J Biomed Biotechnol 2011: 342637, 2011.

35. Nava P, Koch S, Laukoetter MG, Lee WY, Kolegraff K, Capaldo CT, Beeman N, Addis C, Gerner-Smidt K, Neumaier I, Skerra A, Li L, Parkos CA and Nusrat A: Interferon-gamma regulates intestinal epithelial homeostasis through converging beta-catenin signaling pathways. Immunity 32: 392-402, 2010. 